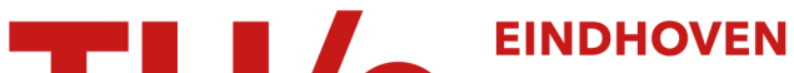 \\ UNIVERSITY OF \\ TECHNOLOGY
}

\section{A queuing model with a randomized depletion of inventory}

\section{Citation for published version (APA):}

Albrecher, H., Boxma, O. J., Essifi, R., \& Kuijstermans, A. C. M. (2017). A queuing model with a randomized depletion of inventory. Probability in the Engineering and Informational Sciences, 31(1), 43-59.

https://doi.org/10.1017/S0269964816000322

\section{Document license: \\ TAVERNE}

DOI:

10.1017/S0269964816000322

Document status and date:

Published: 01/01/2017

\section{Document Version:}

Publisher's PDF, also known as Version of Record (includes final page, issue and volume numbers)

\section{Please check the document version of this publication:}

- A submitted manuscript is the version of the article upon submission and before peer-review. There can be important differences between the submitted version and the official published version of record. People interested in the research are advised to contact the author for the final version of the publication, or visit the $\mathrm{DOI}$ to the publisher's website.

- The final author version and the galley proof are versions of the publication after peer review.

- The final published version features the final layout of the paper including the volume, issue and page numbers.

Link to publication

\section{General rights}

Copyright and moral rights for the publications made accessible in the public portal are retained by the authors and/or other copyright owners and it is a condition of accessing publications that users recognise and abide by the legal requirements associated with these rights.

- Users may download and print one copy of any publication from the public portal for the purpose of private study or research.

- You may not further distribute the material or use it for any profit-making activity or commercial gain

- You may freely distribute the URL identifying the publication in the public portal.

If the publication is distributed under the terms of Article 25fa of the Dutch Copyright Act, indicated by the "Taverne" license above, please follow below link for the End User Agreement:

www.tue.nl/taverne

Take down policy

If you believe that this document breaches copyright please contact us at:

openaccess@tue.nl

providing details and we will investigate your claim. 


\title{
A QUEUEING MODEL WITH RANDOMIZED DEPLETION OF INVENTORY
}

\author{
HANSJÖRG ALBRECHER \\ Department of Actuarial Science, Faculty of Business and Economics, \\ University of Lausanne and Swiss Financial Institute, \\ UNIL-Dorigny, CH-1015 Lausanne, \\ Switzerland \\ Onno Boxma, Rim EsSifI AND RICHARd KUIJSTERMans \\ Department of Mathematics and Computer Science, \\ Eindhoven University of Technology and EURANDOM, \\ P.O. Box 513, 5600 MB Eindhoven, \\ The Netherlands \\ E-mail: o.j.boxma@tue.nl
}

\begin{abstract}
In this paper, we study an $M / M / 1$ queue, where the server continues to work during idle periods and builds up inventory. This inventory is used for new arriving service requirements, but it is completely emptied at random epochs of a non-homogeneous Poisson process, whose rate depends on the current level of the acquired inventory. For several shapes of depletion rates, we derive differential equations for the stationary density of the workload and the inventory level and solve them explicitly. Finally, numerical illustrations are given for some particular examples, and the effects of this depletion mechanism are discussed.
\end{abstract}

Keywords: applied probability; inventory theory; queueing theory; stochastic modelling

\section{INTRODUCTION}

In this paper, we combine queueing and inventory theory. We consider a classical single server queue, but with the special feature that the server keeps working even when there are no customers. It then builds up inventory. This inventory is used for new arriving customers (who are now possibly served instantaneously), but the built-up inventory is removed at random epochs of a non-homogeneous Poisson process, whose rate depends on the current level of the acquired inventory. Our goal is to study the resulting two-sided stochastic model, obtaining both the steady-state workload level (when positive) and the steadystate inventory level (when positive) and their means. That will also yield other interesting performance measures like the probability that a service request is instantaneously satisfied (sojourn time zero). Our model is in particular of interest in situations where the service is homogeneous across customers (e.g., serving means producing some good).

This model obviously is closely related to classical queueing $(M / M / 1)$ and inventory models. A rich literature on queueing systems with inventory was developed in recent years, and with various approaches; but when it comes to computing the stationary distribution 
of inventory and/or workload states (which will interest us in this paper), the topic seems to be less investigated. We would like to mention the book of Sahin [15], and papers like $[6,10,14,16,17]$, which are more oriented toward lost sale problems.

An important inventory model is the one with a basestock policy, in which a server produces products until the inventory has reached a certain basestock level, and in which requests for products arrive according to a Poisson process. If a request cannot be immediately satisfied, it joins a backorder queue. However, that model has a finite basestock level, and hence essentially differs from our model.

A production/inventory-oriented paper that seems related to our study is [12] on sporadic clearing policies. That paper considers a production/inventory system that is continuously filled at a fixed rate and satisfies demands at Poisson epochs. Under the sporadic clearing policy, clearing of all inventory takes place after a random time (which in [12] is independent of the content process). Explicit results are obtained for an expected discounted cost functional. See also [7], which considers the case of exponentially distributed demands, and derives the steady-state inventory level.

Our model is motivated by some recent work in insurance risk theory, where the event of the declaration of bankruptcy was also modeled by an epoch of such a non-homogeneous Poisson process with rate $\omega(x)$ whenever the surplus process of the insurance portfolio is negative (see, e.g., $[2,4]$ ). There are many interesting methodological links between the field of insurance risk theory and queueing (cf. Asmussen and Albrecher [5] for an overview). In the present context, it also turns out that, although the model setups in [2] and the present paper are not identical, the formulas for workload densities and inventory densities of the resulting queueing/inventory model are of a similar complexity as the respective formulas in the risk setting, and we will obtain explicit expressions for those shapes of $\omega(x)$ for which this was also possible in the insurance model in [2].

The rest of the paper is organized as follows. In Section 2, we will introduce the model in detail. Section 3 then works out the respective quantities related to workload and inventory for the case of exponential service requirements. In particular, we consider a constant, a linear and an exponential depletion rate. Finally, Section 4 gives concrete numerical examples and studies the behavior of the considered quantities as a function of the model parameters. Section 5 concludes.

\section{MODEL DESCRIPTION}

Consider the following queueing model. Customers arrive according to a Poisson process with rate $\lambda$. Their service requirements are independent, identically distributed random variables $B_{1}, B_{2}, \ldots$ with distribution $B(\cdot)$ and LST (Laplace-Stieltjes transform) $\beta(\cdot)$. $B$ will denote a generic service requirement. The server works continuously, at a fixed speed which is normalized to 1 - even if there are no service requirements. In the latter case, the server is building up inventory, which can be interpreted as negative workload. At random times, with an intensity $\omega(x)>0$ when the inventory is at level $x>0$, the present inventory is removed, instantaneously reducing the inventory to zero. Put differently, inventory is removed according to a Poisson process with a rate that depends on the amount of inventory present.

Denote the required work per time unit by $\rho:=\lambda \mathbb{E} B$. We assume that $\rho<1$. This ensures that the steady-state workload distribution exists. Let $V_{+}(x), x>0$, denote this steady-state workload distribution and $v_{+}(x)$ its density. During the times in which the inventory level is positive, there is an upward drift $1-\rho$ of that inventory level; but when $\omega(x)>0$ for $x$ sufficiently large, the inventory level will always eventually return to zero, and 


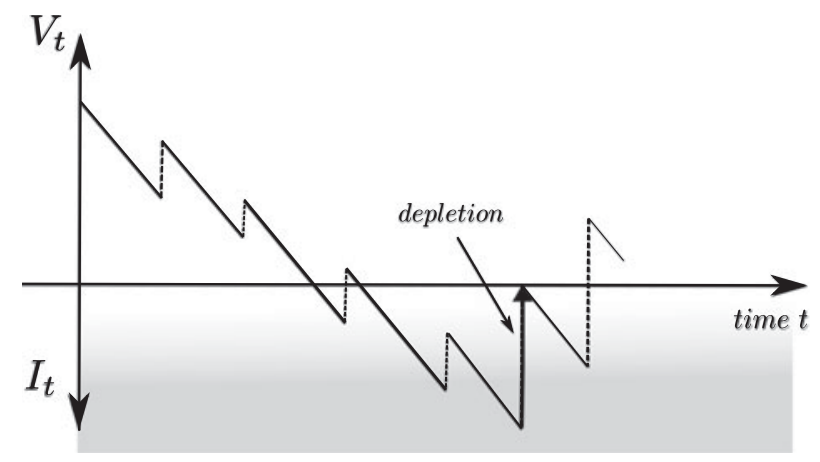

FiguRE 1. Sample path of the workload $V_{t}$ together with the inventory level $I_{t}$.

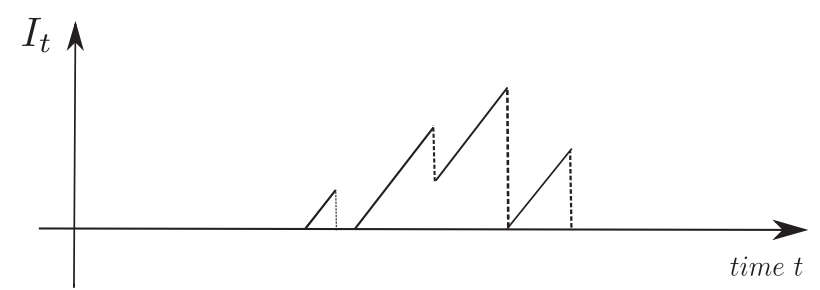

Figure 2. Inventory level $I_{t}$ of Figure 1 displayed separately.

the steady-state inventory distribution will exist. Let $V_{-}(x), x>0$, denote this steady-state inventory distribution and $v_{-}(x)$ its density. Figure 1 displays the workload $V_{t}$ as a function of time $t$ (above the $t$-axis) and the corresponding inventory level $I_{t}$ (below the $t$-axis) in one figure. Figure 2 separately displays the inventory level. Notice that this is also the inventory level corresponding to the production/inventory system with sporadic clearing studied in [12]; but in that paper, clearing occurs according to a fixed rate, and the inventory level distribution is not derived. That distribution is derived in [7], where the case of exponentially distributed demands is considered. It should be noticed that their model is related to our model, in the sense that it is one-sided; in [7] only inventory is considered, there is no queue.

The classical level crossing technique (see, e.g., [9]) yields the following integral equations for the workload and inventory densities:

$$
\begin{aligned}
& v_{+}(x)=\lambda \int_{0}^{x} \mathbb{P}(B>x-y) v_{+}(y) d y+\lambda \int_{0}^{\infty} \mathbb{P}(B>x+y) v_{-}(y) d y, \quad x>0, \\
& v_{-}(x)=\lambda \int_{x}^{\infty} \mathbb{P}(B>y-x) v_{-}(y) d y+\int_{x}^{\infty} \omega(y) v_{-}(y) d y, \quad x>0 .
\end{aligned}
$$

\section{EXPONENTIAL SERVICE REQUIREMENTS}

In this paper, we restrict ourselves to the case of exponential service requirements $\mathbb{P}(B>$ $x)=e^{-\mu x}$. Then (1) and (2) reduce to:

$$
\begin{aligned}
& v_{+}(x)=\lambda e^{-\mu x} \int_{0}^{x} e^{\mu y} v_{+}(y) d y+\lambda e^{-\mu x} \int_{0}^{\infty} e^{-\mu y} v_{-}(y) d y, \quad x>0, \\
& v_{-}(x)=\lambda e^{\mu x} \int_{x}^{\infty} e^{-\mu y} v_{-}(y) d y+\int_{x}^{\infty} \omega(y) v_{-}(y) d y, \quad x>0 .
\end{aligned}
$$


Let $z_{+}(x):=e^{\mu x} v_{+}(x), x>0$, and $z_{-}(x):=e^{-\mu x} v_{-}(x), x>0$, so that (3) and (4) become

$$
\begin{aligned}
& z_{+}(x)=\lambda \int_{0}^{x} z_{+}(y) d y+\lambda \int_{0}^{\infty} z_{-}(y) d y, \quad x>0, \\
& z_{-}(x)=\lambda \int_{x}^{\infty} z_{-}(y) d y+e^{-\mu x} \int_{x}^{\infty} \omega(y) e^{\mu y} z_{-}(y) d y, \quad x>0 .
\end{aligned}
$$

Differentiation of (5) readily leads to

$$
z_{+}(x)=C \lambda e^{\lambda x}, \quad x>0
$$

with $C:=\int_{0}^{\infty} z_{-}(y) d y$, so

$$
v_{+}(x)=C \lambda e^{-(\mu-\lambda) x}, \quad x>0 .
$$

In particular, one has

$$
\int_{0}^{+\infty} v_{+}(x) d x=C \frac{\rho}{1-\rho} .
$$

It is not surprising that $v_{+}(x)$ is, up to a constant, equal to the density of the $M / M / 1$ queue. When the workload becomes positive, it does so via a jump from below the $t$ axis; and (the excess of) that jump is $\exp (\mu)$ distributed. And as long as the workload remains positive, it behaves exactly like the workload in an $M / M / 1$ queue. We later turn to the determination of the missing constant $C=\int_{0}^{\infty} z_{-}(y) d y$. Differentiation of (6) yields:

$$
z_{-}^{\prime}(x)=-\lambda z_{-}(x)-\omega(x) z_{-}(x)-\mu\left[z_{-}(x)-\lambda \int_{x}^{\infty} z_{-}(y) d y\right]
$$

and hence

$$
z_{-}^{\prime \prime}(x)+(\lambda+\mu+\omega(x)) z_{-}^{\prime}(x)+\left(\lambda \mu+\omega^{\prime}(x)\right) z_{-}(x)=0, \quad x>0 .
$$

Below we discuss several choices of the rate function $\omega(\cdot)$ for which (11) can be solved explicitly.

\subsection{Case 1: $\omega(x) \equiv \omega, x>0$}

When $\omega(x) \equiv \omega,(11)$ reduces to:

$$
z_{-}^{\prime \prime}(x)+(\lambda+\mu+\omega) z_{-}^{\prime}(x)+\lambda \mu z_{-}(x)=0, \quad x>0 .
$$

Hence,

$$
z_{-}(x)=C_{1} e^{r_{1} x}+C_{2} e^{r_{2} x}, \quad x>0,
$$

with

$$
r_{1,2}=\frac{1}{2}\left(-(\lambda+\mu+\omega) \mp \sqrt{(\lambda+\mu+\omega)^{2}-4 \lambda \mu}\right)
$$

and finally

$$
v_{-}(x)=C_{1} e^{s_{1} x}+C_{2} e^{s_{2} x}, \quad x>0
$$

with

$$
s_{1,2}=r_{1,2}+\mu=\frac{1}{2}\left(-(\lambda-\mu+\omega) \mp \sqrt{(\lambda+\mu+\omega)^{2}-4 \lambda \mu}\right) .
$$

The constants $C$ (cf. (8)), $C_{1}$ and $C_{2}$ can be determined as follows. First observe that $s_{2}>$ 0 , so that $C_{2}$ must be zero. Next observe that $C=\int_{0}^{\infty} z_{-}(y) d y=C_{1} /-r_{1}=C_{1} /\left(\mu-s_{1}\right)$. 
Finally, use that

$$
\int_{0}^{\infty} v_{+}(x) d x+\int_{0}^{\infty} v_{-}(x) d x=1
$$

We thus find that

$$
C_{1}=\frac{(\mu-\lambda)\left(\mu-s_{1}\right) s_{1}}{\mu\left(s_{1}-\mu+\lambda\right)}
$$

and

$$
C=\frac{(\mu-\lambda) s_{1}}{\mu\left(s_{1}-\mu+\lambda\right)}
$$

Finally, the solution reads

$$
\begin{aligned}
v_{-}(x)= & \frac{(\mu-\lambda)\left(\lambda-\mu+\omega+\sqrt{(\lambda+\mu+\omega)^{2}-4 \lambda \mu}\right)\left(\lambda+\mu+\omega+\sqrt{(\lambda+\mu+\omega)^{2}-4 \lambda \mu}\right)}{2 \mu\left(-\lambda+\mu+\omega+\sqrt{(\lambda+\mu+\omega)^{2}-4 \lambda \mu}\right)} \\
& \times e^{(1 / 2)\left(-\lambda+\mu-\omega-\sqrt{(\lambda+\mu+\omega)^{2}-4 \lambda \mu}\right)}, \quad x>0 ; \\
v_{+}(x)= & \frac{(\mu-\lambda)\left(\lambda-\mu+\omega+\sqrt{(\lambda+\mu+\omega)^{2}-4 \lambda \mu}\right)}{\mu\left(-\lambda+\mu+\omega+\sqrt{(\lambda+\mu+\omega)^{2}-4 \lambda \mu}\right)} \lambda e^{(\lambda-\mu) x}, \quad x>0 .
\end{aligned}
$$

The mean inventory level is in this case

$$
\begin{aligned}
\mathbb{E}[I] & =\int_{0}^{\infty} x v_{-}(x) d x \\
& =\frac{2(\mu-\lambda)\left(\sqrt{(\lambda+\mu+\omega)^{2}-4 \lambda \mu}+\lambda+\mu+\omega\right)}{\mu\left(\sqrt{(\lambda+\mu+\omega)^{2}-4 \lambda \mu}+\lambda-\mu+\omega\right)\left(\sqrt{(\lambda+\mu+\omega)^{2}-4 \lambda \mu}-\lambda+\mu+\omega\right)} .
\end{aligned}
$$

The mean workload level is calculated as

$$
\mathbb{E}[X]=\int_{0}^{\infty} x v_{+}(x) d x=\frac{\lambda\left(\sqrt{(\lambda+\mu+\omega)^{2}-4 \lambda \mu}+\lambda-\mu+\omega\right)}{\mu(\mu-\lambda)\left(\sqrt{(\lambda+\mu+\omega)^{2}-4 \lambda \mu}-\lambda+\mu+\omega\right)},
$$

and the probability of finding no inventory in stationarity is

$$
\int_{0}^{\infty} v_{+}(x) d x=\frac{\lambda\left(\sqrt{(\lambda+\mu+\omega)^{2}-4 \lambda \mu}+\lambda-\mu+\omega\right)}{\mu\left(\sqrt{(\lambda+\mu+\omega)^{2}-4 \lambda \mu}-\lambda+\mu+\omega\right)} .
$$

Conversely, the probability for an arriving customer to be served immediately is given by

$$
\int_{0}^{\infty} v_{-}(x) d x=1-\frac{\lambda\left(\sqrt{(\lambda+\mu+\omega)^{2}-4 \lambda \mu}+\lambda-\mu+\omega\right)}{\mu\left(\sqrt{(\lambda+\mu+\omega)^{2}-4 \lambda \mu}-\lambda+\mu+\omega\right)} .
$$

Note that for $\omega \rightarrow \infty$ (immediate depletion of the inventory, i.e. no inventory) we retrieve the classical result that the probability to arrive in an idle period is $1-\lambda / \mu$. On the other hand, for $\omega=0$ we obtain $\int_{0}^{\infty} v_{-}(x) d x=1$, as one should expect because the drift in the 
workload process is downward. The probability that due to the inventory an arriving customer can leave the system immediately again (i.e., his service requirement was available in the inventory, sojourn time 0) is

$$
\int_{0}^{\infty} v_{-}(x) \mathbb{P}(B<x) d x=\int_{0}^{\infty} v_{-}(x)\left(1-e^{-\mu x}\right) d x=\frac{2(\mu-\lambda)}{\sqrt{(\lambda+\mu+\omega)^{2}-4 \lambda \mu}-\lambda+\mu+\omega} .
$$

\subsection{Case 2: $\omega(x)=a x, x>0, a>0$}

Differentiating Eq. (4), when $\omega(x)=a x$, one gets the following equation for $v_{-}$:

$$
v_{-}^{\prime}(x)+(\lambda+a x) v_{-}(x)-\lambda \mu e^{\mu x} \int_{x}^{+\infty} e^{-\mu y} v_{-}(y) d y=0,
$$

in particular, plugging $x=0$ in relation (24), we obtain

$$
C=\frac{\lambda v_{-}(0)+v_{-}^{\prime}(0)}{\lambda \mu}
$$

Now, differentiating the expression in Eq. (24), the function $v_{-}$satisfies the following secondorder differential equation

$$
v_{-}^{\prime \prime}(x)+(\lambda-\mu+a x) v_{-}^{\prime}(x)+a(1-\mu x) v_{-}(x)=0 .
$$

Introduce the function $\theta(x)=v_{-}(x) e^{\left(a x^{2} / 2\right)+\lambda x}$. The function $v_{-}$is a solution of Eq. (26) if and only if the function $\theta$ is a solution of the following second-order differential equation:

$$
\theta^{\prime \prime}(x)-(\lambda+\mu+a x) \theta^{\prime}(x)+\lambda \mu \theta(x)=0 .
$$

One can check that $\theta$ is a solution of Eq. (27) if and only if

$$
\theta(x)=\mathcal{J}\left(\tilde{a}, \tilde{b}, \frac{a}{2}\left(x+\frac{\lambda+\mu}{a}\right)^{2}\right)
$$

where $\tilde{a}=-(\lambda \mu / 2 a)$ and $\tilde{b}=1 / 2$ and $\mathcal{J}(\tilde{a}, \tilde{b}, \cdot)$ is a solution of the degenerate hypergeometric equation:

$$
\xi y^{\prime \prime}(\xi)+(\tilde{b}-\xi) y^{\prime}(\xi)-\tilde{a} y(\xi)=0 .
$$

According to [11, p. 322] and [13, p. 143], Eq. (28) has two standard solutions denoted by $\xi \mapsto M(\tilde{a}, \tilde{b}, \xi)$ and $\xi \mapsto U(\tilde{a}, \tilde{b}, \xi)$, the so-called Kummer functions. Provided that $\tilde{b} \notin$ $\{-1,-2, \ldots\}$, the function $\xi \mapsto M(\tilde{a}, \tilde{b}, \xi)$ is given by

$$
M(\tilde{a}, \tilde{b}, \xi)=\sum_{0}^{+\infty} \frac{(\tilde{a})_{s}}{(\tilde{b})_{s} s !} \xi^{s} \quad \text { for all } \xi \in \mathbb{C}
$$

where $(c)_{s}=c(c+1) \cdots(c+s-1)$. The function $\xi \mapsto U(\tilde{a}, \tilde{b}, \xi)$ is uniquely determined by the property $U(\tilde{a}, \tilde{b}, \xi) \sim \xi^{-a}$ when $\xi$ goes to $+\infty$. In our case $(\tilde{b}=1 / 2)$, one has

$$
U(\tilde{a}, \tilde{b}, \xi)=\frac{\Gamma(1 / 2)}{\gamma(1 / 2-\lambda \mu / 2 a)} M\left(-\frac{\lambda \mu}{2 a}, \frac{1}{2}, \xi\right)+\Gamma\left(-\frac{1}{2}\right) \xi^{1 / 2} M\left(-\frac{\lambda \mu}{2 a}+\frac{1}{2}, \frac{3}{2}, \xi\right) .
$$


The general solution of Eq. (28) is given by:

$$
y(\xi)=C_{3} M(\tilde{a}, \tilde{b}, \xi)+C_{4} \xi^{1-\tilde{b}} M(\tilde{a}-\tilde{b}+1,2-\tilde{b}, \xi), \quad \text { where } \quad C_{3}, C_{4} \in \mathbb{R} .
$$

Thanks to relation (29), the general solution of Eq. (28) can also be expressed in the following way:

$$
y(\xi)=K_{1} M(\tilde{a}, \tilde{b}, \xi)+K_{2} U(\tilde{a}, \tilde{b}, \xi), \quad \text { where } \quad K_{1}, K_{2} \in \mathbb{R} .
$$

Then, in our case, one gets:

$$
\mathcal{J}\left(-\frac{\lambda \mu}{2 a}, \frac{1}{2}, \xi\right)=K_{1} M\left(-\frac{\lambda \mu}{2 a}, \frac{1}{2}, \xi\right)+K_{2} U\left(-\frac{\lambda \mu}{2 a}, \frac{1}{2}, \xi\right), \quad \text { where } \quad K_{1}, K_{2} \in \mathbb{R} .
$$

Remembering that for all $x \geq 0$, one has

$$
v_{-}(x)=e^{-(a / 2) x^{2}-\lambda x} \mathcal{J}\left(-\frac{\lambda \mu}{2 a}, \frac{1}{2}, \frac{a}{2}\left(x+\frac{\lambda+\mu}{a}\right)^{2}\right),
$$

one obtains for all $x \geq 0$,

$$
\begin{aligned}
v_{-}(x)= & e^{-(a / 2) x^{2}-\lambda x} \\
& \times\left[K_{1} M\left(-\frac{\lambda \mu}{2 a}, \frac{1}{2}, \frac{a}{2}\left(x+\frac{\lambda+\mu}{a}\right)^{2}\right)+K_{2} U\left(-\frac{\lambda \mu}{2 a}, \frac{1}{2}, \frac{a}{2}\left(x+\frac{\lambda+\mu}{a}\right)^{2}\right)\right],
\end{aligned}
$$

where $K_{1}, K_{2} \in \mathbb{R}$. Now the plan is to determine the constants $K_{1}$ and $K_{2}$. To do so, we will use the well-known asymptotic behavior of the functions $\xi \mapsto M(\tilde{a}, \tilde{b}, \xi)$ and $\xi \mapsto U(\tilde{a}, \tilde{b}, \xi)$. We will distinguish two cases.

Case 1: $\tilde{a}=-\lambda \mu / 2 a \notin \mathbb{Z}$, that is $\lambda \mu / 2 a \notin \mathbb{N}$. Denote by $\nu:=\lambda \mu / a$. In this case, according to $[11$, p. 323$]$, one knows that $M(\tilde{a}, \tilde{b}, \xi) \sim\left[e^{\xi} \xi^{\tilde{a}-\tilde{b}}\right] /[\Gamma(\tilde{a})]$, when $\xi$ goes to $+\infty$, and so,

$$
\begin{aligned}
& M\left(-\frac{\lambda \mu}{2 a}, 1 / 2, \frac{a}{2}\left(x+\frac{\lambda+\mu}{a}\right)^{2}\right) \\
& \quad \sim \frac{(a / 2)^{a-(1 / 2)}}{\Gamma(-\lambda \mu / 2 a)} e^{(a / 2)(x+(\lambda+\mu) / a)^{2}}\left(x+\frac{\lambda+\mu}{a}\right)^{-(\lambda \mu / a)-1}, \quad \text { when } x \rightarrow+\infty .
\end{aligned}
$$

Now, according to [11, p. 322], one knows that

$$
U(\tilde{a}, \tilde{b}, \xi) \sim \xi^{-\tilde{a}} \text { when } \xi \rightarrow+\infty,
$$

and then

$$
U\left(-\frac{\lambda \mu}{2 a}, \frac{1}{2}, \frac{a}{2}\left(x+\frac{\lambda+\mu}{a}\right)^{2}\right) \sim a^{\lambda \mu / 2 a}\left(x+\frac{\lambda+\mu}{a}\right)^{\lambda \mu / a}, \quad \text { when } x \rightarrow+\infty .
$$

Since the function $v_{-}$is integrable, Eq. (33) and relations (34), (36) imply that the constant $K_{1}$ should be necessarily equal to zero and then, one has

$$
v_{-}(x)=K_{2} e^{-\frac{a}{2} x^{2}-\lambda x} U\left(-\frac{\nu}{2}, \frac{1}{2}, \frac{a}{2}\left(x+\frac{\lambda+\mu}{a}\right)^{2}\right), \quad \forall x \geq 0 .
$$


Now coming back to Eq. (25), one obtains

$$
C=\frac{K_{2}(\lambda+\mu) U^{\prime}\left(-(\nu / 2), 1 / 2,(\lambda+\mu)^{2} / 2 a\right)}{\lambda \mu} .
$$

On the other hand, using the relation $\int_{0}^{+\infty} v_{+}(x) d x+\int_{0}^{+\infty} v_{-}(x) d x=1$, and Eqs. (9), (33), one gets

$$
C \frac{\rho}{1-\rho}+K_{2} \int_{0}^{+\infty} e^{-(a / 2) x^{2}-\lambda x} U\left(-\frac{\nu}{2}, 1 / 2, a / 2\left(x+\frac{\lambda+\mu}{a}\right)^{2}\right) d x=1 .
$$

Finally, relations (38) and (39) allow us to determine the constants $K_{2}$ and $C$.

In particular, if $\nu$ is odd, that is $\nu=2 n+1$, where $n \in \mathbb{N}$, then from [11, p. 325], one knows that

$$
U(\tilde{a}, \tilde{b}, z)=z^{1-\tilde{b}} U(\tilde{a}-\tilde{b}+1,2-\tilde{b}, z), \quad z \in \mathbb{C} .
$$

For $x \geq 0$, set

$$
z^{2}=\frac{a}{2}\left(x+\frac{\lambda+\mu}{a}\right)^{2}
$$

relation (40) implies

$$
U\left(-\frac{\nu}{2}, \frac{1}{2}, \frac{a}{2}\left(x+\frac{\lambda+\mu}{a}\right)^{2}\right)=U\left(-\frac{\nu}{2}, \frac{1}{2}, z^{2}\right)=z U\left(-\frac{\nu}{2}+\frac{1}{2}, \frac{3}{2}, z^{2}\right) .
$$

For $m \in \mathbb{Z}$, let $H_{m}(\cdot)$ be the Hermite polynomial of order $m$ (see $[1$, p. 775]) given by the formula $H_{m}(x)=(-1)^{m} e^{(1 / 2) x^{2}}(d / d x)^{m}\left[e^{(-1 / 2) x^{2}}\right]$. According to [11, p. 328] (notice that we are using in this paper the notation $H_{m}$, which in [11] is denoted by $H e_{m}$ ), for $z \in \mathbb{C}$, one has

$$
U\left(-\frac{\nu}{2}+\frac{1}{2}, \frac{3}{2}, z^{2}\right)=2^{-(\nu / 2)} z^{-1} H_{\nu}(\sqrt{2} z) .
$$

Combining relations (37), (41) and (42), one gets

$$
v_{-}(x)=2^{-(\nu / 2)} K_{2} e^{-(a / 2) x^{2}-\lambda x} H_{\nu}\left(\sqrt{a} x+\frac{\lambda+\mu}{\sqrt{a}}\right), \quad \forall x \geq 0 .
$$

Case 2: $\lambda \mu / 2 a=n \in \mathbb{N}$. In this case, thanks to Eq. (30), one has, for all $x \geq 0$,

$$
\begin{aligned}
v_{-}(x)= & e^{-(a / 2) x^{2}-\lambda x}\left[K_{1} M\left(-n, \frac{1}{2}, \frac{a}{2}\left(x+\frac{\lambda+\mu}{a}\right)^{2}\right)+K_{2} \sqrt{\frac{a}{2}}\left(x+\frac{\lambda+\mu}{a}\right)\right. \\
& \left.\times M\left(-n+\frac{1}{2}, \frac{3}{2}, \frac{a}{2}\left(x+\frac{\lambda+\mu}{a}\right)^{2}\right)\right],
\end{aligned}
$$

where $K_{1}, K_{2} \in \mathbb{R}$. According to [11, p. 328], for $z \in \mathbb{C}$, one has

$$
M\left(-n, \frac{1}{2}, \frac{z^{2}}{2}\right)=(-1)^{n} \frac{n !}{(2 n) !} 2^{n} H_{2 n}(\sqrt{2} z)=(-1)^{\nu / 2} \frac{(\nu / 2) !}{\nu !} 2^{\frac{\nu}{2}} H_{\nu}(\sqrt{2} z)
$$


and

$$
z^{1 / 2} M\left(-n+\frac{1}{2}, \frac{3}{2}, z\right) \sim \frac{z^{-n-1} e^{z}}{\Gamma(-n+(1 / 2))}, \quad \text { when } z \rightarrow+\infty .
$$

Therefore, the integrability condition satisfied by the function $v_{-}$implies that the constant $K_{2}$ must be zero in this case, and one obtains

$$
v_{-}(x)=(-1)^{\nu / 2} \frac{(\nu / 2) !}{\nu !} 2^{\nu / 2} K_{1} e^{-(a / 2) x^{2}-\lambda x} H_{\nu}\left(\sqrt{a} x+\frac{\lambda+\mu}{\sqrt{a}}\right), \quad x \geq 0 .
$$

Following the same line of reasoning as previously, one can find two linear equations involving the unknowns $C$ and $K_{1}$ and then determine them.

\subsection{Case 3: $\omega(x)=e^{b x}, x>0, b>0$}

If $\omega(x)=e^{b x},(11)$ reduces to

$$
z_{-}^{\prime \prime}(x)+\left(\lambda+\mu+e^{b x}\right) z_{-}^{\prime}(x)+\left(\lambda \mu+b e^{b x}\right) z_{-}(x)=0, \quad x>0 .
$$

Below we show how, via a substitution, one can arrive at exactly the same Kummer differential equation (28) as we found for the case $\omega(x)=a x$ (there we had to use other substitutions). Substituting $z_{-}(x)=e^{-\left(e^{b x} / b\right)} g(x)$, we get

$$
g^{\prime \prime}(x)+\left(\lambda+\mu-e^{b x}\right) g^{\prime}(x)+\left(\lambda \mu-(\lambda+\mu) e^{b x}\right) g(x)=0, \quad x>0 .
$$

Now introduce $u(x):=e^{b x} / b$ and substitute $g(x)=e^{-\mu x} y(u(x))=(b u(x))^{-(\mu / b)} y(u(x))$ in (49). This leads to the Kummer differential equation

$$
u y^{\prime \prime}(u)+\left(\frac{\lambda-\mu}{b}+1-u\right) y^{\prime}(u)-\frac{\lambda}{b} y(u)=0, \quad u>0
$$

with solution (cf. [1, Ch.13])

$$
y(u)=C_{5} U\left(\frac{\lambda}{b}, 1+\frac{\lambda-\mu}{b}, u\right)+C_{6} F_{1,1}\left(\frac{\lambda}{b}, 1+\frac{\lambda-\mu}{b}, u\right),
$$

where this time we use the notation $F_{1,1}(., .,$.$) for the hypergeometric function, instead of$ $M(., .,$.$) , to be consistent with [2]$.

Hence,

$$
g(x)=e^{-\mu x}\left(C_{5} U\left(\frac{\lambda}{b}, 1+\frac{\lambda-\mu}{b}, \frac{e^{b x}}{b}\right)+C_{6} F_{1,1}\left(\frac{\lambda}{b}, 1+\frac{\lambda-\mu}{b}, \frac{e^{b x}}{b}\right)\right),
$$

and subsequently

$$
\begin{aligned}
& z_{-}(x)=e^{-\left(e^{b x} / b\right)} e^{-\mu x}\left(C_{5} U\left(\frac{\lambda}{b}, 1+\frac{\lambda-\mu}{b}, \frac{e^{b x}}{b}\right)+C_{6} F_{1,1}\left(\frac{\lambda}{b}, 1+\frac{\lambda-\mu}{b}, \frac{e^{b x}}{b}\right)\right), \\
& v_{-}(x)=e^{-\left(e^{b x} / b\right)}\left(C_{5} U\left(\frac{\lambda}{b}, 1+\frac{\lambda-\mu}{b}, \frac{e^{b x}}{b}\right)+C_{6} F_{1,1}\left(\frac{\lambda}{b}, 1+\frac{\lambda-\mu}{b}, \frac{e^{b x}}{b}\right)\right)
\end{aligned}
$$

with $C_{5}, C_{6} \in \mathbb{R}$. In addition, (8) still holds. The constants $C, C_{5}$ and $C_{6}$ follow from the conditions (15),

$$
C=\frac{z_{-}^{\prime}(0)+(\lambda+\mu+1) z_{-}(0)}{\lambda \mu}
$$


and $\lim _{x \rightarrow \infty} v_{-}(x)=0$. We have, from $(34)$,

$$
\begin{aligned}
\lim _{x \rightarrow \infty} e^{-\left(e^{b x} / b\right)} U\left(\frac{\lambda}{b}, 1+\frac{\lambda-\mu}{b}, \frac{e^{b x}}{b}\right) & =\lim _{x \rightarrow \infty} e^{-\left(e^{b x} / b\right)}\left(\frac{e^{b x}}{b}\right)^{-(\lambda / b)} \\
& =\lim _{x \rightarrow \infty} e^{-\left(e^{b x} / b\right)}\left(\frac{1}{b}\right)^{-(\lambda / b)} e^{-\lambda x}=0 .
\end{aligned}
$$

Furthermore, from (35),

$$
\begin{aligned}
\lim _{x \rightarrow \infty} e^{-\left(e^{b x} / b\right)} F_{1,1}\left(\frac{\lambda}{b}, 1+\frac{\lambda-\mu}{b}, \frac{e^{b x}}{b}\right) & =\lim _{x \rightarrow \infty} \frac{\Gamma(1+(\lambda-\mu) / b)}{\Gamma(\lambda / b)}\left(\frac{1}{b}\right)^{(\mu / b)-1} e^{(\mu-b) x} \\
& = \begin{cases}+\infty, & \text { if } \mu>b, \\
1, & \text { if } \mu=b, \\
0, & \text { if } \mu<b .\end{cases}
\end{aligned}
$$

Consequently, if $\mu \geq b$, then $C_{6}=0$ (in contrast to the risk model in [2], we cannot a priori exclude the case $\mu<b$ here, but we nevertheless assume $\mu \geq b$ for convenience of the present context). Then the over-all solution reads:

$$
\left\{\begin{array}{l}
v_{-}(x)=e^{-\frac{e^{b x}}{b}} C_{5} U\left(\frac{\lambda}{b}, 1+\frac{\lambda-\mu}{b}, \frac{e^{b x}}{b}\right) ; \\
v_{+}(x)=C \lambda e^{(\lambda-\mu) x}
\end{array}\right.
$$

with the constants $C$ and $C_{5}$ still to be determined. From (55)

$$
C=C_{5} \frac{e^{-(1 / b)}(b U((\lambda / b), 1+(\lambda-\mu) / b, 1 / b)-U(1+(\lambda / b), 2+(\lambda-\mu) / b, 1 / b))}{\mu b} .
$$

From (15) we then obtain

$$
\begin{aligned}
C_{5}= & \left(\int_{0}^{\infty} e^{-\left(e^{b y} / b\right)} U\left(\frac{\lambda}{b}, 1+\frac{\lambda-\mu}{b}, \frac{e^{b y}}{b}\right) d y\right. \\
& \left.+\frac{e^{-(1 / b)}(b U(\lambda / b, 1+(\lambda-\mu) / b, 1 / b)-U(1+(\lambda / b), 2+(\lambda-\mu) / b, 1 / b))}{\mu b} \frac{\lambda}{\mu-\lambda}\right)^{-1},
\end{aligned}
$$

and finally, for $x>0$,

$$
v_{+}(x)=\left(\frac{(\mu b / \lambda) e^{1 / b} \int_{0}^{\infty} e^{-\left(e^{b y} / b\right)} U\left(\lambda / b, 1+(\lambda-\mu) / b,\left(e^{b y} / b\right)\right) d y}{b U(\lambda / b, 1+(\lambda-\mu) / b, 1 / b)-U(1+\lambda / b, 2+(\lambda-\mu) / b, 1 / b)}+\frac{1}{\mu-\lambda}\right)^{-1} e^{(\lambda-\mu) x} .
$$

\section{NUMERICAL RESULTS}

In this subsection, we give some numerical illustrations for the case $\omega(x) \equiv \omega$. 


\subsection{Mean inventory level}

The mean inventory level $\mathbb{E}[I]=\int_{0}^{\infty} x v_{-}(x) d x$ is given in Figure 3 as a function of the Poisson rate $\lambda$ (keeping the other parameters fixed) when the function $\omega$ is constant $(\omega(x)=$ $\omega>0)$, linear $(\omega(x)=a x)$ and exponential $\left(\omega(x)=e^{b x}\right)$. Figure 4 depicts $\mathbb{E}[I]$ as a function of the exponential rate $\mu$ of the service requirements.

In Figure 3, we see that if $\lambda$ increases and approaches $\mu$, the mean inventory level decreases (in fact, almost linearly). If $\rho=\lambda / \mu$ approaches 1 , it is hardly possible to build up an inventory. In Figure 4, we see that if $\mu$ increases, the mean inventory level also increases. This is obvious, as the service requirements become smaller. For the displayed cases, $\omega(x)=e^{b x} \geq 1+b x>a x$, for all $x \geq 0$ and accordingly $\mathbb{E} I$ for $\omega(x)=e^{b x}$ is smaller than $\mathbb{E} I$ for $\omega(x)=a x$.

\subsection{Mean workload}

Next, consider the mean workload $\int_{0}^{\infty} x v_{+}(x) d x$. Figures 5-7 depict the mean workload as a function of $\lambda$ for different values of the parameters $\mu, a, \omega$ and $b$ when the function $\omega$ is constant $(\omega(x)=\omega>0)$, linear $(\omega(x)=a x)$ and exponential $\left(\omega(x)=e^{b x}\right)$. Similarly, Figures $8-10$ depict the mean workload as a function of $\mu$.

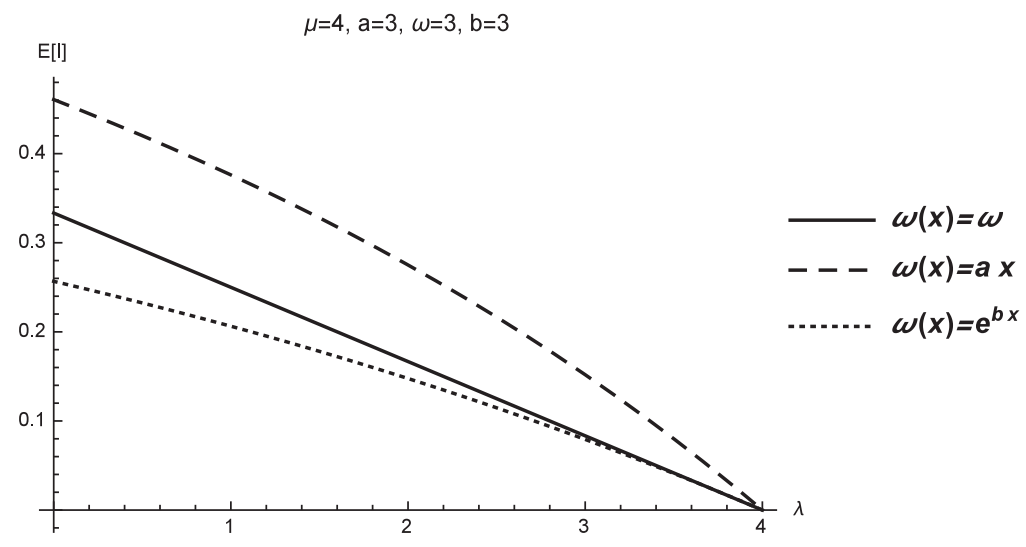

Figure 3. Mean inventory level, with $\mu=4, a=3, \omega=3$ and $b=3$.

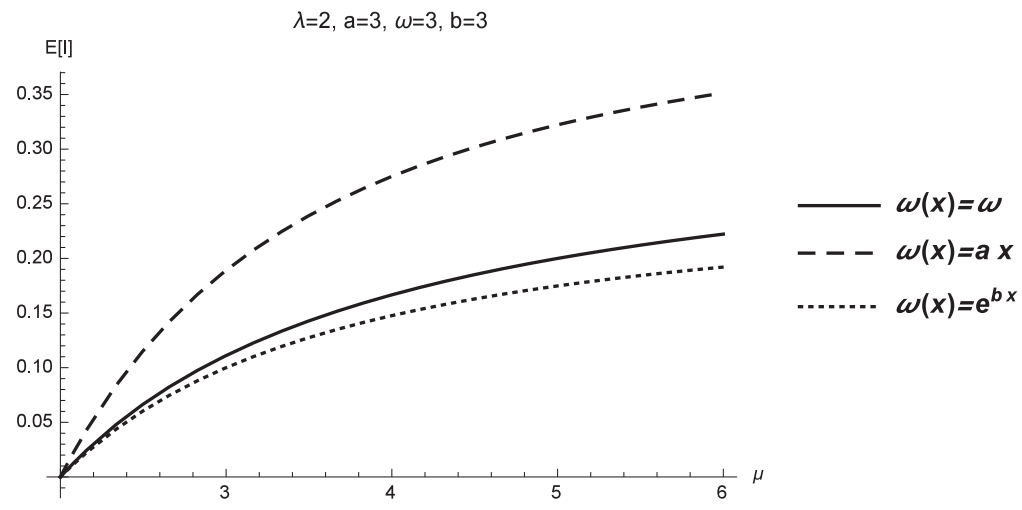

Figure 4. Mean inventory level, with $\lambda=2, a=3, \omega=3$ and $b=3$. 


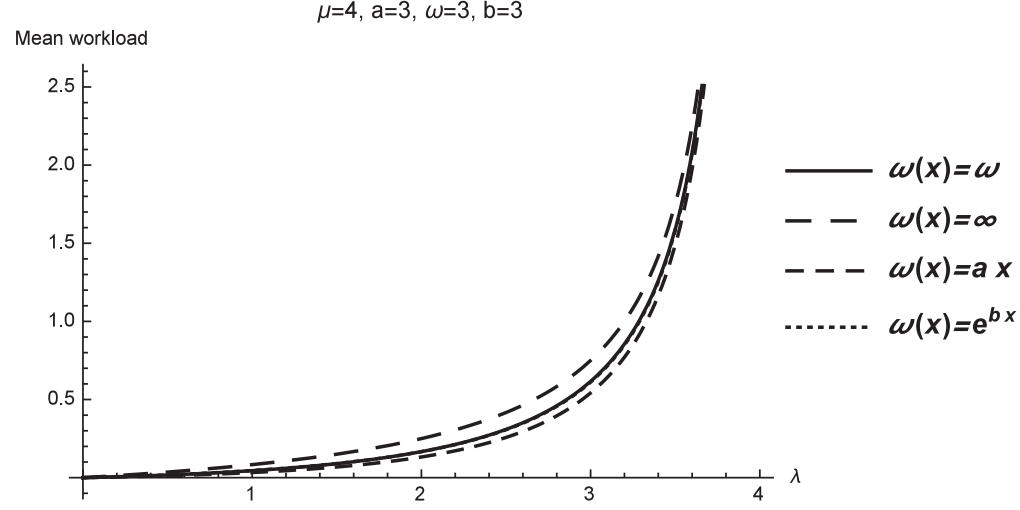

FiguRE 5. Mean workload, with $\mu=4, a=3, \omega=3$ and $b=3$.

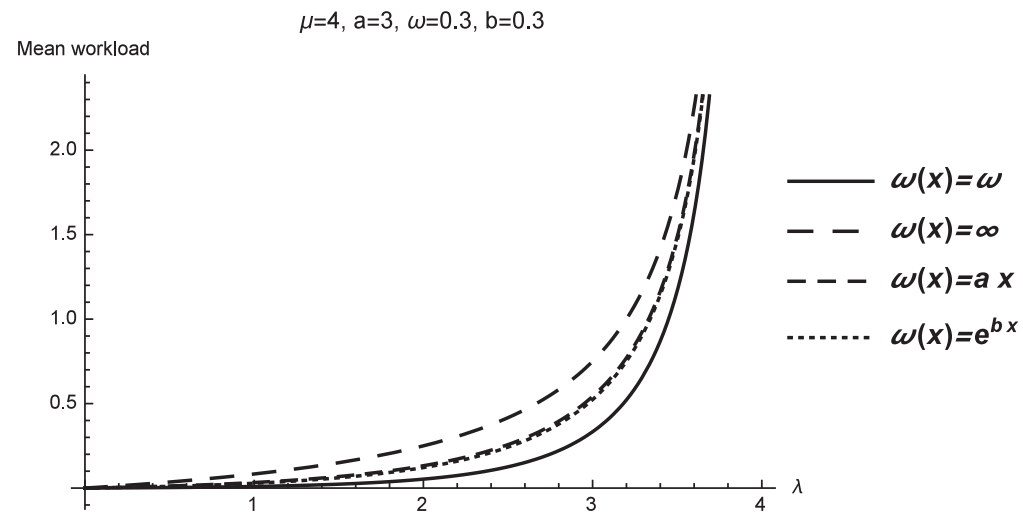

FiguRE 6. Mean workload, with $\mu=4, a=3, \omega=0.3$ and $b=0.3$.

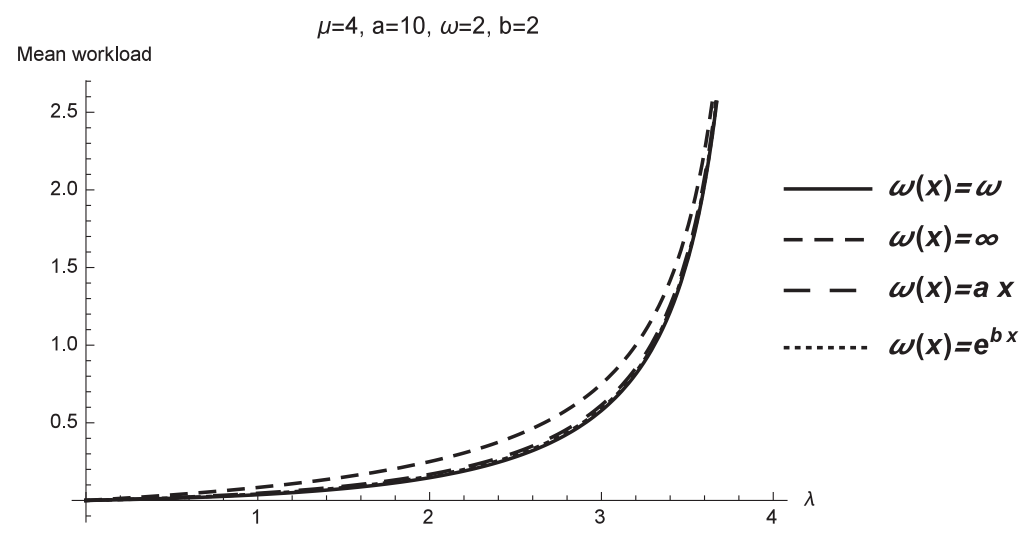

FiguRE 7. Mean workload, with $\mu=4, a=10, \omega=2$ and $b=2$. 


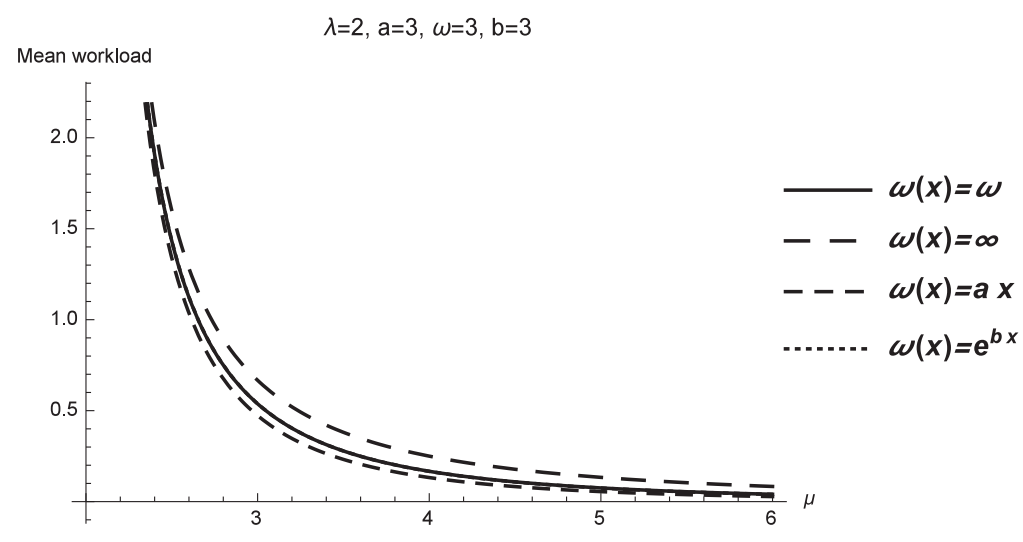

Figure 8. Mean workload, with $\lambda=2, a=3, \omega=3$ and $b=3$.

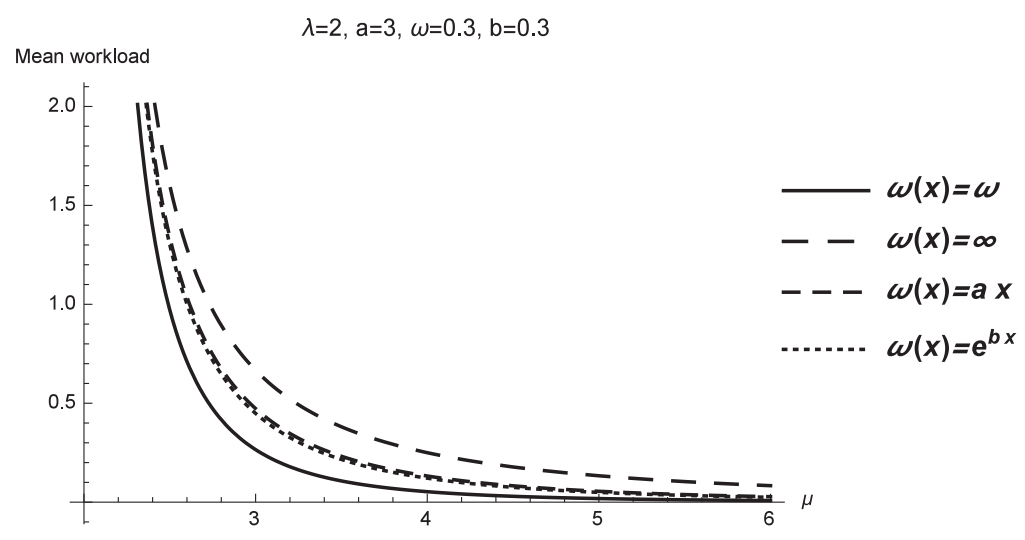

Figure 9. Mean workload, with $\lambda=2, a=3, \omega=0.3$ and $b=0.3$.

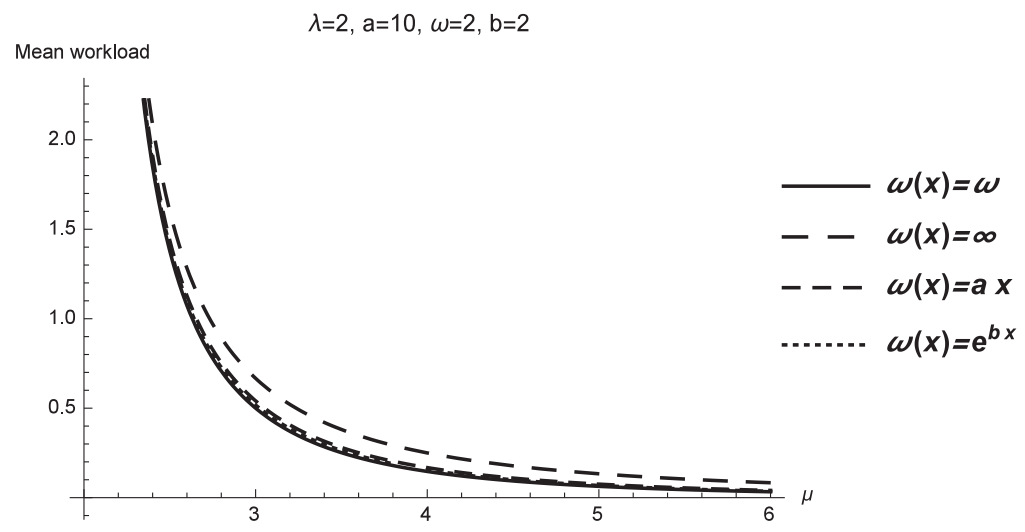

Figure 10. Mean workload, with $\lambda=2, a=10, \omega=2$ and $b=2$. 
From the figures below, we see that the various $\omega$-choices lead to similar results in heavy traffic. This makes sense because in this case it is hardly relevant what happens during periods of a positive inventory; those periods are too rare. In the case $a=b=\omega=3$, one has $\omega(x)=e^{3 x} \geq 1+3 x>3 x$, and it makes sense that the mean workload for $\omega(x)=e^{3 x}$ is larger than the mean workload for $\omega(x)=3 x$; of course $\omega(x)=+\infty$ gives the largest mean workload.

\subsection{Probability of having no inventory}

Figures 11-13 depict the probability of finding no inventory in the stationary state $\int_{0}^{+\infty} v_{+}(x) d x$ as a function of $\lambda$ for different values of the parameters $\mu, a, \omega$ and $b$ when the function $\omega$ is constant $(\omega(x)=\omega>0)$, linear $(\omega(x)=a x)$ and exponential $\left(\omega(x)=e^{b x}\right)$.

Similarly, Figures 14-16 depict the probability of finding no inventory in the stationary state as a function of $\mu$ instead of $\lambda$. The resulting shapes are quite intuitive.

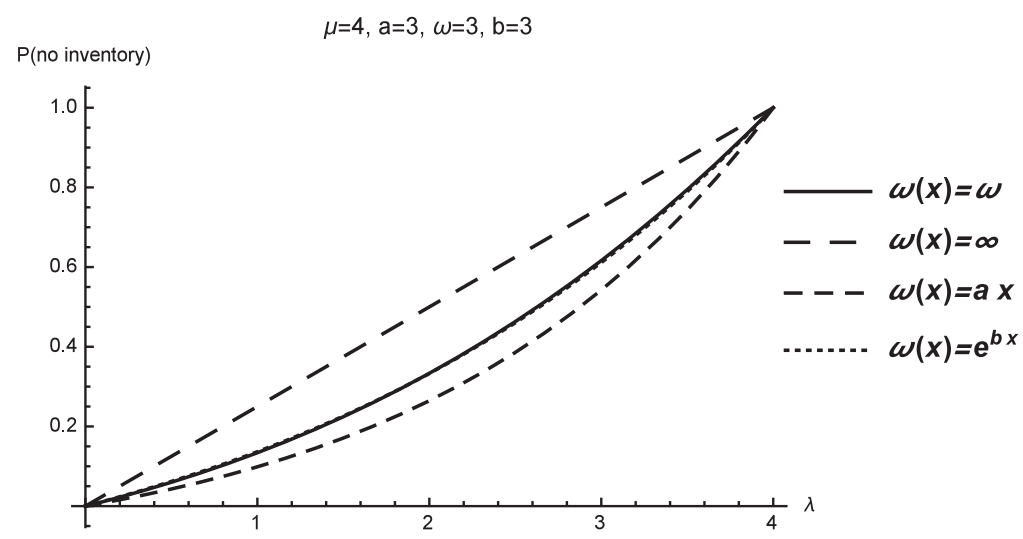

Figure 11. Probability of no inventory, with $\mu=4, a=3, \omega=3$ and $b=3$.

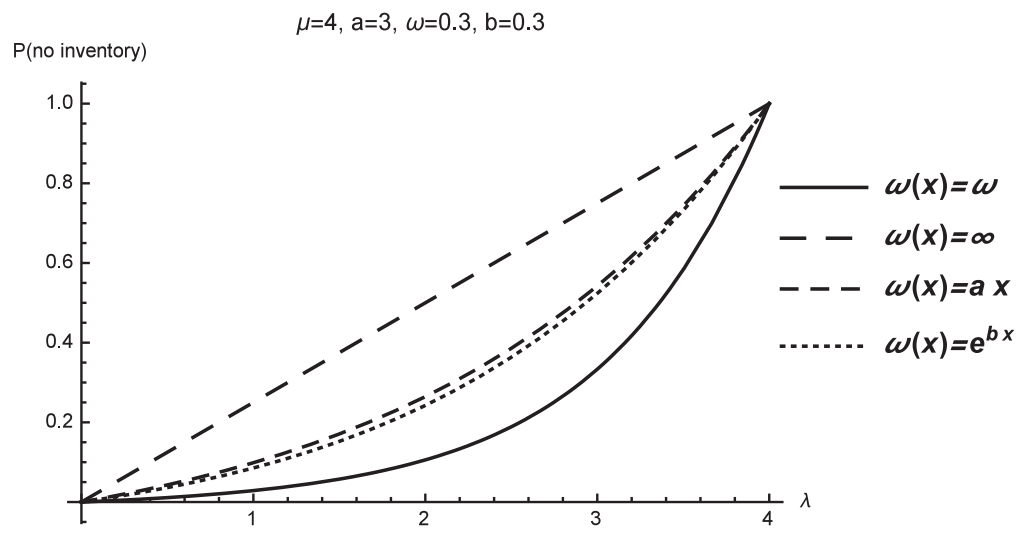

Figure 12. Probability of no inventory, with $\mu=4, a=3, \omega=0.3$ and $b=0.3$. 


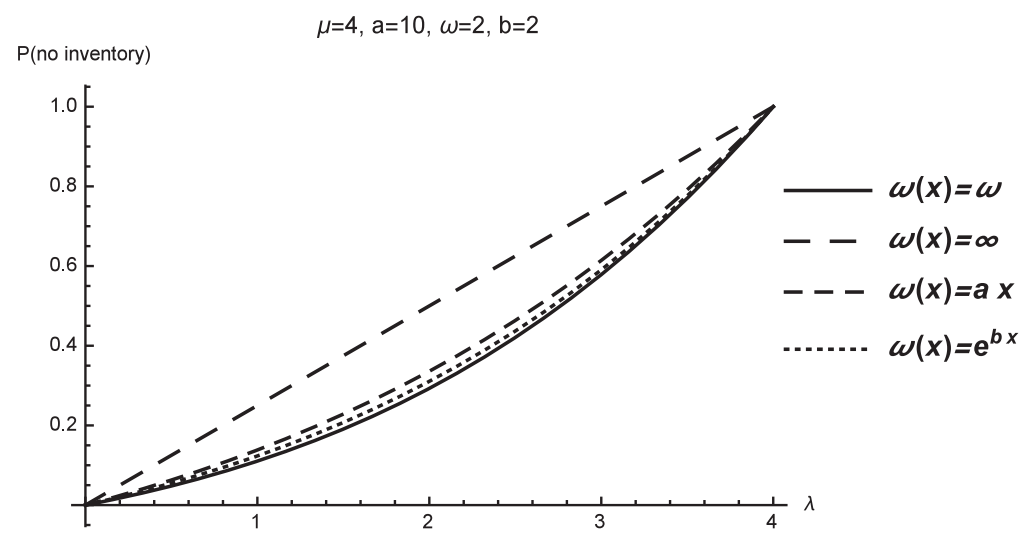

Figure 13. Probability of no inventory, with $\mu=4, a=10, \omega=2$ and $b=2$.

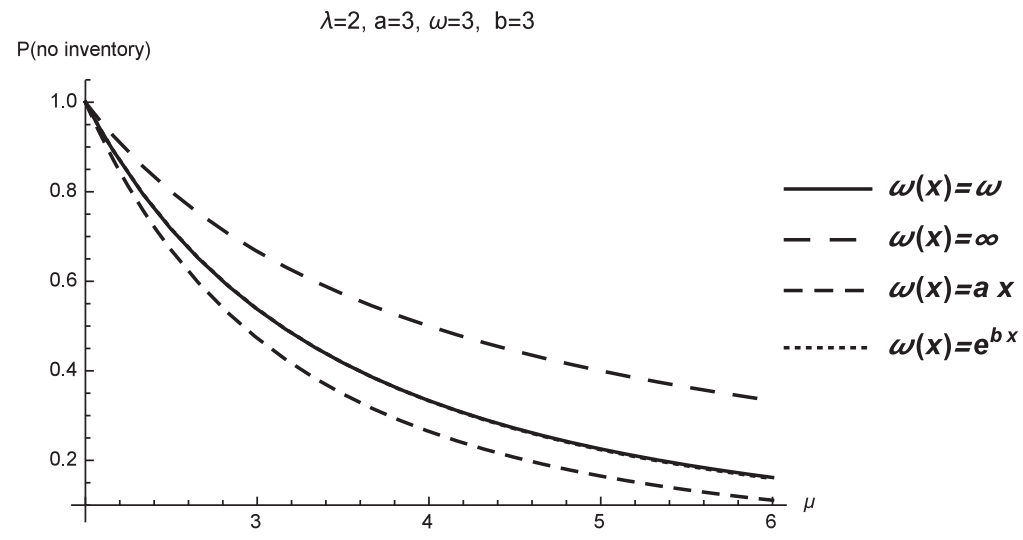

Figure 14. Probability of no inventory, with $\lambda=2, a=3, \omega=3$ and $b=3$.

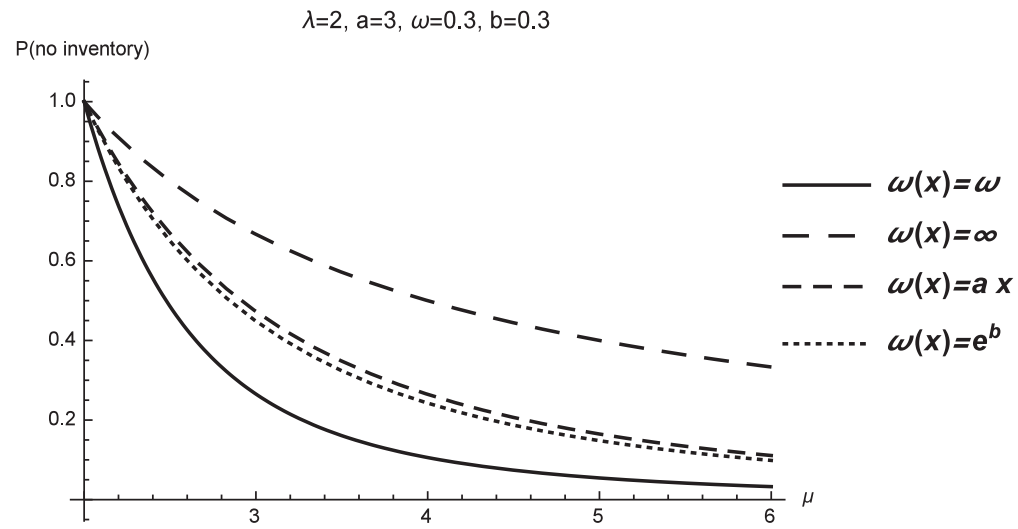

Figure 15. Probability of no inventory, with $\lambda=2, a=3, \omega=0.3$ and $b=0.3$. 


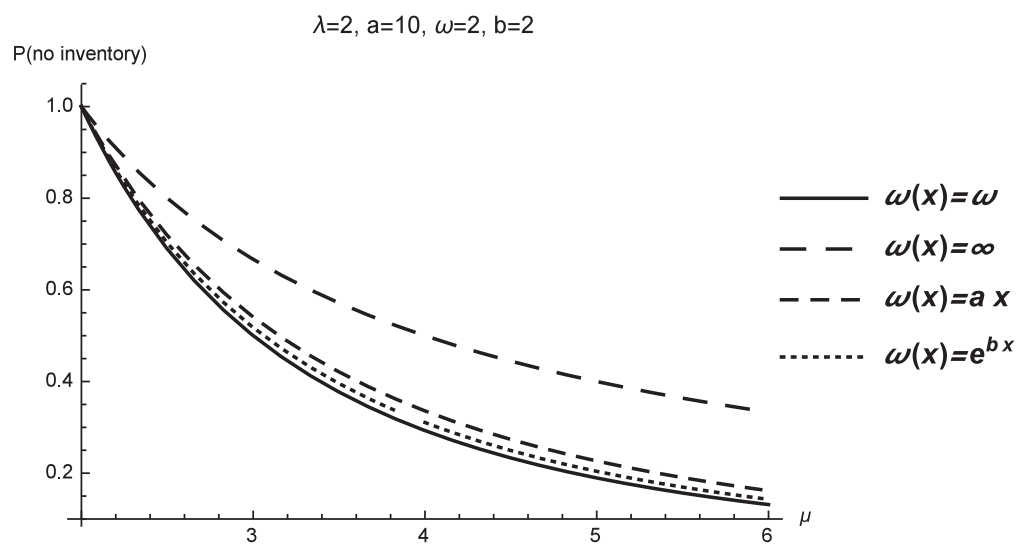

Figure 16. Probability of no inventory, with $\lambda=2, a=10, \omega=2$ and $b=2$.

\section{CONCLUSION}

In this paper, we considered a queueing model with inventory, where the inventory is depleted at random times according to a Poisson process with surplus-dependent intensity. For a number of intensity shapes this leads to explicit formulas, and for constant depletion rate the expressions become particularly simple.

There are a number of directions for possible future research. Such a random depletion mechanism with its simple formulas may serve as an approximation of depletion mechanisms at deterministic time points. In fact, if the depletion mechanism according to a homogeneous Poisson process is extended to a renewal process with Erlang-distributed inter-occurrence times, an analysis in the spirit of [3] may still be possible in the present setup. We leave this extension for future research. For surplus-dependent Poisson rates, we have seen that the expressions, although explicit, quickly become quite involved. One alternative may be to consider piecewise constant upper and lower bounds for $\omega(x)$ and determine upper and lower bounds for the quantities under consideration through smooth-pasting of tractable expressions at the respective layer boundaries (see [2] for such a mechanism in the risk theory context).

It may be also worthwhile to explore the following idea. Application of the transformation

$$
\theta(x)=e^{\lambda x+\int_{0}^{x} \omega(y) d y} v_{-}(x)
$$

to (4) gives

$$
\theta^{\prime \prime}(x)-(\lambda+\mu+\omega(x)) \theta^{\prime}(x)+\lambda \mu \theta(x)=0 .
$$

Notice that this transformation was applied below Eq. (26) for the case $\omega(x)=a x$, and is closely related to the transformation applied below Eq. (48) for the case $\omega(x)=e^{b x}$. It could be very interesting to solve Eq. (58) for other $\omega(x)$, or to find a function transformation that even provides a solution to (58) for general $\omega(\cdot)$.

Another direction for future research is optimization. One might like to choose $\omega(x)$ in such a way that the profit (for example, income from serving customers and from selling inventory at depletion epochs, minus waiting costs and holding costs) is maximized. One might also try to optimally choose the server speed during the periods in which there is no customer; an analysis of the model with different server speeds during periods with and without customers does not pose any problem. 
An important extension is to allow generally distributed service times. The analysis then becomes considerably more involved, but for $\omega(x) \equiv \omega$ an approach via Wiener-Hopf factorization appears possible; see, for example, [8].

\section{Acknowledgements}

The research of Hansjörg Albrecher was supported by the Swiss National Science Foundation Project 200020_143889. The research of Onno Boxma was supported by the NETWORKS project, which is being funded by the Dutch government. The research of Rim Essifi was supported by the ERC CritiQueue program.

\section{References}

1. Abramowitz, M. \& Stegun, I.A. (1964). Handbook of mathematical functions with formulas, graphs and mathematical tables. New York, NY: Dover Publications.

2. Albrecher, H. \& Lautscham, V. (2013). From ruin to bankruptcy for compound Poisson surplus processes. ASTIN Bulletin 43: 213-243.

3. Albrecher, H., Cheung, E.C.K., \& Thonhauser, S. (2013). Randomized observation periods for the compound Poisson risk model: the discounted penalty function. Scandinavian Actuarial Journal 6: $424-452$.

4. Albrecher, H., Gerber, H.U., \& Shiu, E.S.W. (2011). The optimal dividend barrier in the Gamma-Omega model. European Actuarial Journal 1: 43-55.

5. Asmussen, S. \& Albrecher, H. (2010). Ruin probabilities. Hackensack, NJ: World Scientific.

6. Berman, O. \& Kim, E. (2001). Dynamic order replenishment policy in internet-based supply chains. Mathematical Methods of Operations Research 53: 371-390.

7. Berman, O., Parlar, M., Perry, D., \& Posner, M.J.M. (2005). Production/clearing models under continuous and sporadic review. Methodology and Computing in Applied Probability 7: 203-224.

8. Boxma, O.J., Essifi, R., \& Janssen, A.J.E.M. (2015). A queueing/inventory and an insurance risk model. To appear in Journal of Applied Probability

9. Doshi, B.T. (1992). Level crossing analysis of queues. In U.N. Bhat \& I.V. Basawa (eds.), Queueing and related models, Oxford: Clarendon Press, pp. 3-33

10. Mohebbi, E. \& Posner, M.J.M. (1998). A continuous-review inventory system with lost sales and variable lead time. Naval Research Logistics 45: 259-278.

11. Olver, F.W.J., Lozier, D.W., Boisvert, R.F., \& Clark, C.W. (2010). NIST handbook of mathematical functions. NIST, New York, NY: Cambridge University Press.

12. Perry, D., Stadje, W., \& Zacks, S. (2005). Sporadic and continuous clearing policies for a production/inventory system under an $M / G$ demand process. Mathematics of Operations Research 30: 354-368.

13. Polyanin, A.D., \& Zaitsev, V.F. (2003). Handbook of exact solutions for ordinary differential equations, 2nd ed., Boca Raton, FL: CRC Press.

14. Saffari, M., Asmussen, S., \& Haji, R. (2013). The M/M/1 queue with inventory, lost sale, and general lead times. Queueing Systems 75: 65-77.

15. Sahin, I. (1990). Regenerative inventory systems, operating characteristics and optimization, New York, NY: Springer.

16. Schwarz, M. \& Daduna, H. (2006). Queueing systems with inventory management with random lead times and with backordering. Mathematical Methods of Operations Research 64: 383-414.

17. Schwarz, M., Sauer, C., Daduna, H., Kulik, R., \& Szekli, R. (2006). M/M/1 queueing systems with inventory. Queueing Systems 54: 55-78. 